\title{
Site-specific bacterial chromosome engineering mediated by IntA integrase from Rhizobium etli
}

\author{
Rogelio Hernández-Tamayo ${ }^{1}$, Gonzalo Torres-Tejerizo ${ }^{1,2}$, Susana Brom and David Romero ${ }^{\text {** }}$
}

\begin{abstract}
Background: The bacterial chromosome may be used to stably maintain foreign DNA in the mega-base range. Integration into the chromosome circumvents issues such as plasmid replication, stability, incompatibility, and copy number variance. The site-specific integrase IntA from Rhizobium etli CFN42 catalyzes a direct recombination between two specific DNA sites: attA and attD (23 bp). This recombination is stable. The aim of this work was to develop a $R$. etli derivative that may be used as recipient for the integration of foreign DNA in the chromosome, adapting the IntA catalyzed site-specific recombination system.

Results: To fulfill our aim, we designed a Rhizobium etli CFN42 derivative, containing a "landing pad" (LP) integrated into the chromosome. The LP sector consists of a green fluorescent protein gene under the control of the lacZ promoter and a spectinomycin resistance gene. Between the lacZ promoter and the GFP gene we inserted an IntA attachment site, which does not affect transcription from the lac promoter. Also, a mobilizable donor vector was generated, containing an attA site and a kanamycin resistance gene; to facilitate insertion of foreign DNA, this vector also contains a multicloning site. There are no promoters flanking the multicloning site. A biparental mating protocol was used to transfer the donor vector into the landing pad strain; insertion of the donor vector into the landing pad sector via IntA-mediated attA X attA recombination thereby interrupted the expression of the green fluorescent protein, generating site-specific cointegrants. Cointegrants were easily recognized by screening for antibiotic sensitivity and lack of GFP expression, and were obtained with an efficiency of $6.18 \%$.

Conclusions: Integration of foreign DNA in Rhizobium, lacking any similarity with the genome, can be easily achieved by IntA-mediated recombination. This protocol contains the mating and selection procedures for creating and isolating integrants.
\end{abstract}

Keywords: Tyrosine recombinase, Chromosomal integration, Site-specific recombination

\section{Background}

A critical factor for genome engineering of complex, novel characteristics in bacteria, is the efficient introduction and stabilization of large segments of DNA lacking similarity to the host genome. Plasmid-based strategies are of limited use in this regard, due to problems of plasmid instability and unforeseen consequences generated by copy number variations. Site-specific recombination mediated by integrases can neatly circumvent these

\footnotetext{
* Correspondence: dromero@ccg.unam.mx

'Programa de Ingeniería Genómica, Centro de Ciencias Genómicas,

Universidad Nacional Autónoma de México, Apartado Postal 565-A, 62210

Cuernavaca, Morelos, Mexico

Full list of author information is available at the end of the article
}

problems, providing a means for effective integration of large DNA fragments in specific genomic locations. Tyrosine recombinases are one of the two classes of proteins that catalyze site-specific recombination [1]. Most of the members of this family are highly efficient in promoting recombination, leading to excision, integration or both events, although in the case of bidirectional tyrosine recombinases, with contrasting efficiencies. Unlike homologous recombination, which requires extensive similarity between recombining sequences, tyrosine recombinases catalyze recombination between substrates sharing limited sequence identity. Sequence identity normally spans a short region (8-15 bp) where DNA exchange occurs. The properties of high efficiency and 
reduced size of target sequences for recombination, make integrases excellent tools for the manipulation of DNA. Integrases are also amenable to a variety of genetic modifications that may enable the production of custom integrases with selectivity for novel target sequences [2].

Systems for chromosomal integration of foreign DNA mediated by tyrosine integrases were devised as early as the 1990s for Escherichia coli [3], Staphylococcus aureus [4] and Actinobacteria [5, 6]. All of them employ special plasmid vectors harboring the corresponding recombinase recognition sequence (att or attachment site), where foreign DNA can be cloned. Upon introduction into target cells expressing the cognate integrase, site-specific integration occurs via the endogenous attachment site.

The two most widely used systems for in vivo recombination based on tyrosine recombinases are Cre-loxP and Flp-frt [7]. These systems have a proven efficacy in a variety of biological systems. However, both promote excision more readily than integration. Moreover, given the complex manipulations needed for generation for strains with new features, there is a growing demand of novel systems that employ different recombination systems.

In a previous study we characterized the function of the Rhizobium etli IntA site-specific recombinase, through a combination of in vivo and in vitro assays [8]. IntA belongs to the tyrosine-recombinase family. It allows cointegration of plasmids $\mathrm{p} 42 \mathrm{a}$ and the symbiotic plasmid via site-specific recombination between attachment regions in $R$. etli. The results described previously revealed that IntA is able to catalyze both integration $\left(2.4 \times 10^{-5}\right)$ and excision $\left(2.1 \times 10^{-6}\right)$ events in vivo [8]. The recombination requires the presence of a characteristic $23 \mathrm{bp}$ sequence (the att sector) harboring two arms of a palindrome plus a divergent central region [8]. The sequence of attA (TCCGATAAGCATTACTTATCGGA) differs by a single nucleotide (shown in italics) from $a t t D$; this difference does not affect recombination efficiency with IntA, since attA X attA, attA X attD and $a t t D \mathrm{X} a t t D$ recombination are all equally likely [8].

In the present study, we took advantage of the high efficiency and specificity of integration afforded by this system, to construct an integration system for $R$. etli based on site-specific recombination via IntA integrase. This system allows integration of large DNA segments, in a manner independent of homologous recombination, into predefined sectors in the genome of $R$. etli.

\section{Results and discussion}

$R$. etli has two IntA-dependent recombination sites, att $A$ present in plasmid pRetCFN42a and attD on plasmid pRetCFN42d [9]. Aiming to introduce a supernumerary att site on the chromosome, we modified a previous construction, that contained a green fluorescent protein gene under the control of the lac $Z$ promoter and a spectinomycin resistance gene [10]. This construction was modified by inserting an IntA att site between the lac $Z$ promoter and the GFP gene. This insertion does not affect transcription from the lac $Z$ promoter (see Methods); the whole region was called the "landing pad" (LP) sector (Fig. 1a). For insertion of the LP sector into the chromosome, a region was chosen, where insertion of additional sequences most likely does not interfere with essential activities of Rhizobium etli. Previous genomic analyses revealed a non-coding region in the chromosome, located between bases 1108084 and 1108560; this region is flanked by converging transcription units corresponding to loci RHE_CH01055 and RHE_CH01056 [11]. To facilitate insertion of the LP sector, a chromosomal segment (477 bp, corresponding to the non-coding region) was amplified using primers (Table 1). Insertion of the LP sector in the middle of this chromosomal segment (see Methods) provided extensions of $221 \mathrm{bp}$ (5'side) and 249 bp (3'side), homologous to the chromosome. Insertion of the LP sector into the chromosomal region was readily done by allelic exchange (see Methods and Fig. 1a).

To verify that insertion of the LP sector had occurred in the appropriate chromosomal region, fluorescent derivatives (Fig. 2a) were subjected to PCR amplifications with primer pairs chr_left_out-Plac-out and chr_right_out-CasNot-Ter-out. For both reactions, one of the members of the primer pair is complementary to sequences of the LP sector, while the other is complementary to the neighboring genomic region. As expected, PCR products were detected with both primer pairs (Fig. 2b), ascertaining that the LP sector was inserted in the appropriate chromosomal region. To verify the absence of gross modifications in the plasmid content of the strain carrying the LP sector, plasmid size and content were verified by the Eckhardt in-gel lysis technique [12] as modified by Hynes [13]. No alterations were detected in the plasmid profile (Fig. 2c).

To evaluate if the LP sector can be used as an acceptor of IntA-mediated in vivo recombination, we employed plasmid pK18 mob att $\Delta$ plac (see Methods and Fig. 1b). This plasmid is a modification of the suicide vector pK18 mob containing an attA site, a kanamycin resistance gene, and lacking the plac $Z$ promoter; it also harbors a MCS, where defined segments can be easily introduced. In all our experiments the IntA activity was provided from the intA gene encoded in plasmid pRetCFN42a, present in the LP strain. Plasmid pK18 mob att $\Delta$ plac was mobilized by biparental matings, from an $E$. coli donor to the $R$. etli LP strain. Upon entrance of the plasmid into the LP strain, IntA-mediated recombination may occur with any of three targets: the attA 
A Landing Pad (LP sector)

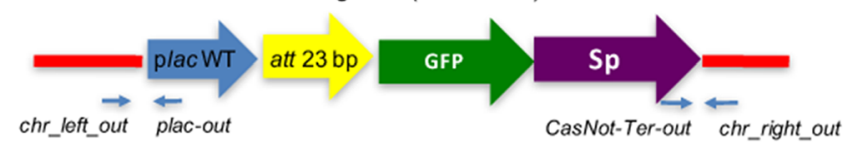

B

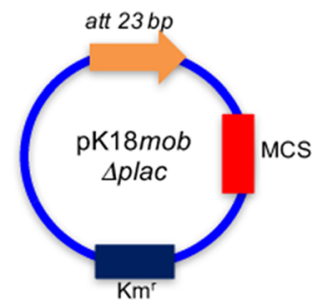

C

Integration of pK18mob $\Delta$ plac on the LP sector

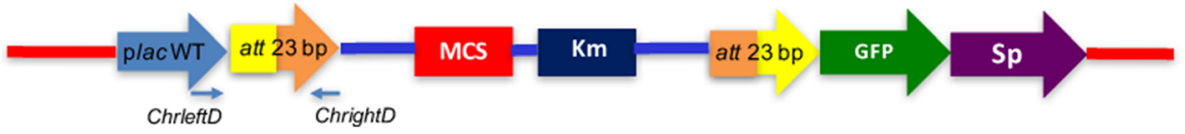

Fig. 1 Diagram of the IntA site-specific integration approach. a A novel region (the Landing Pad or LP sector) was inserted into the chromosome of $R$. etli. This region comprises a new attA region, flanked by a lac promoter and a promoterless green fluorescent protein (GFP) gene; and a spectinomycin resistance gene with its own promoter. $\mathbf{b}$ Mobilizable kanamycin-resistant donor vector (pK18 mob att $\Delta$ plac) containing an attD site and a Multi Cloning site (MCS). The plac promoter was removed from the donor vector as described in Methods. c Predicted structure of integrants of pK18 mob att $\triangle$ plac into the LP sector. Note that integration of the donor vector by attA X attD recombination abolishes transcription of the GFP gene, leading to nonfluorescent colonies. In panels a and $\mathbf{c}$, the location of oligonucleotide primers useful to verify insertion, are indicated as arrows below the appropriate locations

Table 1 Oligonucleotides used in this work

\begin{tabular}{|c|c|c|c|}
\hline Name & Sequence $^{a}$ & Genome location ${ }^{\mathrm{b}}$ & Source or reference \\
\hline chr_left_in_hind & AAAAAAGCTTTCCCGGCTCCGACAG & $1108084 \mathrm{Chr}$ & This work \\
\hline chr_right_in_eco & AAAAGAATTCCCGGTGTCTGCTTCCA & $1108560 \mathrm{Chr}$ & This work \\
\hline chr_left_out & CGGAACACCGGATCTCA & $1107995 \mathrm{Chr}$ & This work \\
\hline chr_right_out & CGTGCCCGCTTTTGTC & $1108840 \mathrm{Chr}$ & This work \\
\hline Plac-out & CGCGGAACCCCTATTTG & & 10 \\
\hline CasNot-Ter-out & CCGTCTGTGATGGCTTCC & & 10 \\
\hline UpattA1 & CTGGATCCCAAAGGTCGCTCCTGAAT & $2252 \mathrm{p} 42 \mathrm{a}$ & 8 \\
\hline LwattA1 & CGAATTCATITTCCCGAGAATAACATG & 2687 p42a & 8 \\
\hline$L w 23 b p$ & CGGATCCTCCGATAAGCATTACTTATCGGACTTAAGT & $5765 \mathrm{p} 42 \mathrm{~d}$ & 8 \\
\hline Up23bp & CGGATCCTCCGATAAGCATTACTTATCGGACTTAAGT & 5743 p42d & 8 \\
\hline M13 reverse & CAGGAAACAGCTATGAC & & ThermoFisher Scientific \\
\hline LwattD1 & CGAATTCCGGCGTTTGCATCTCGTTA & $5875 \mathrm{p} 42 \mathrm{~d}$ & 8 \\
\hline ChrleftD & CTGGATCCTCCGATAAGTAA & & This work \\
\hline ChrightD & CGAATTCTGATTTGCGGGCG & & This work \\
\hline G18/w & CGGATCCTCCGATAAGCATTACTTATCGGACTTAAGT & attG18mob & This work \\
\hline G18up & GCCTAGGAGGCTATTCGTAATGAATAGCCTGAATTCA & attG18mob & This work \\
\hline Link-PstxNot & AGCGGCCGCTTGCA & NA & \\
\hline
\end{tabular}

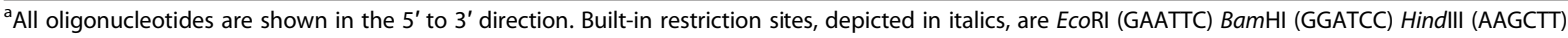
Notl (GCGGCCGC)

'The location is indicated by the first 5' nucleotide and the replicon where the sequence is located. Accession numbers are p42a (NC_007762), p42d (NC_004041), Chr (NC_007761) of R. etli. NA, not applicable (NA) 


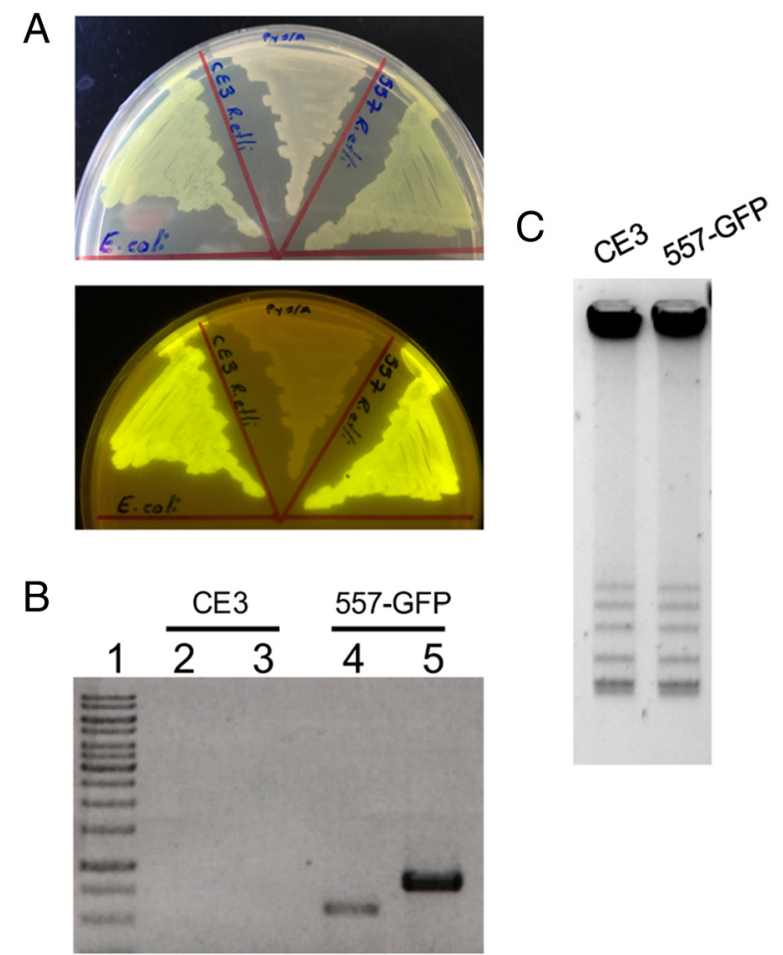

Fig. 2 Characterization of the R. etli LP strain (557). a Visualization of selected strains under white light (top) and UV light (bottom). Strains, in a clockwise order were: E. coli harboring plasmid pRG11 (Landing Pad); R. etli wild type (CE3); R. etli 557 (LP strain). b PCR reactions verifying that integration occurred in the desired chromosomal site. Lanes 2 and 4 are PCR reactions with primers chr_left_out and Plac-out, while lanes 3 and 5 contain PCR reactions with primers CasNot-Ter-out and chr_right_out. The strains analyzed are indicated in the top of the panel. c Plasmid profile of the same strains analyzed in (a) and (b)

located in plasmid pRetCFN42a, the attD from plasmid pRetCFN42d, or the attA present in the chromosomal LP sector. Insertions of $\mathrm{pK} 18$ mob att $\Delta$ plac in the chromosomal LP sector disrupt transcription of the GFP gene coming from the lac $Z$ promoter (Fig. 1c). Thus, insertions in the LP sector can be easily detected by a simple screen for kanamycin and spectinomycin resistant, nonfluorescent derivatives.

Upon conjugative transfer of pK18 mob att $\Delta$ plac to the $R$. etli LP strain, kanamycin resistant integrants were detected at a frequency of $1.4 \times 10-4$ per recipient cell. Individual integrants were picked and visualized under UV light; representative results are shown in Fig. 3. A total of 1019 integrants (coming from five independent experiments) were analyzed for GFP expression; of these, $63(6.18 \%)$ were nonfluorescent, thus indicating insertions in the LP sector. The remaining integrants were probably inserted in the att sites located on pRetCFN42a or pRetCFN42d. Augmented integration into plasmids in comparison to the chromosome was

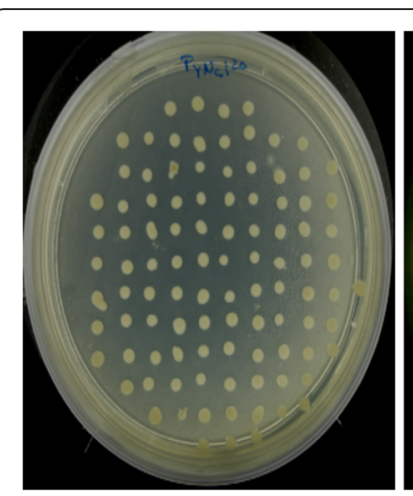

White light

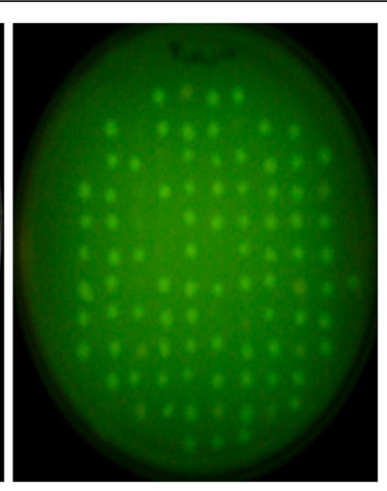

Ultraviolet light
Fig. 3 Isolation of integrants. Visualization of random integrants resulting from integration of pK18 mob att $\Delta$ plac into $R$. etli 557. Note the presence of a relatively high frequency of nonfluorescent colonies, indicating integration of pK18 mob att $\Delta$ plac into the LP sector

expected, given the higher copy number of these replicons, estimated at two for pRetCFN42d and four for pRetCFN42a, with respect to the chromosome (D. Romero, unpublished data).

To verify the site of insertion of each class of integrants, both, nonfluorescent kanamycin and spectinomycin resistant derivatives, as well as fluorescent, kanamycin and spectinomycin resistant integrants, were subjected to PCR analysis with a set of primers that distinguish among insertions in the LP sector (ChrleftD and ChrightD), pRetCFN42a (LwattA1 and M13 reverse), or pRetCFN42d (LwattD1 and M13 reverse). As shown in Fig. 4, nonfluorescent, kanamycin and spectinomycin resistant derivatives rendered a PCR product with primers specific for the LP sector, but not with primers specific for pRetCFN42a or pRetCFN42d (Fig. 4c). In contrast, fluorescent, kanamycin and spectinomycin resistant integrants occurred either in pRetCFN42a or pRetCFN42d, depending of the integrant. Location of the insertions was further verified using Southern blot hybridizations of the plasmid profiles with pK18 mob as probe (Fig. 4a and b). The six nonfluorescent, spectinomycin resistant derivatives analyzed in this way presented insertion on the LP sector; from five fluorescent, spectinomycin resistant integrants that were screened, three revealed integration of pK18 mob att $\triangle$ plac on pRetCFN42a, while in the remaining two, insertion occurred on pRetCFN42d.

To evaluate the stability of integrants of pK18 mob att $\Delta$ plac in the LP sector, one integrant was serially subcultured in the absence of antibiotics, for a period corresponding to 21 generations. Dilutions were plated on solid media without antibiotics. A total of 10000 colonies were screened for GFP expression, interestingly, all of them remained nonfluorescent. 

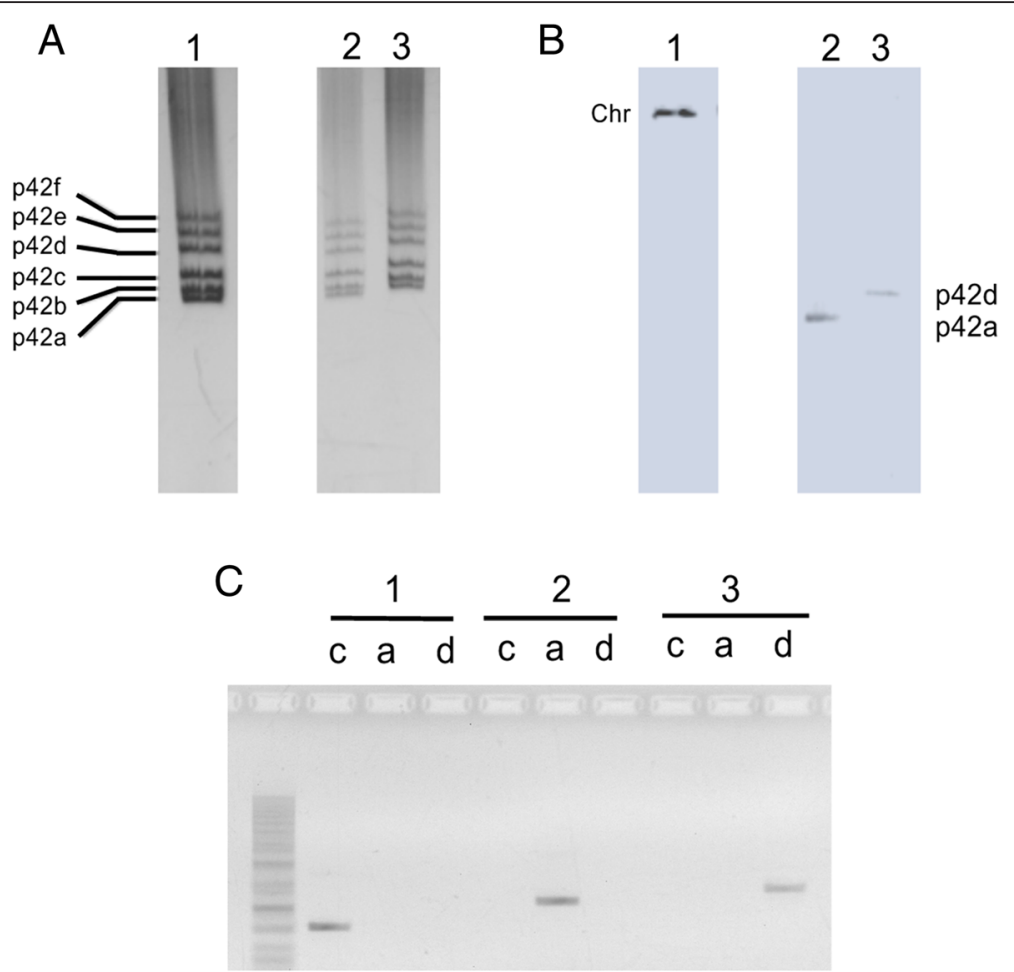

Fig. 4 Analyses of selected cointegrates. All panels: lanes or blocks marked 1 correspond to a nonfluorescent kanamycin resistant cointegrate, while lanes or blocks 2 and 3 correspond to different fluorescent kanamycin resistant cointegrates. a Plasmid profiles of selected strains, stained with ethidium bromide. Plasmid identification is depicted at the left of the panel. $\mathbf{b}$ Southern blots of the corresponding plasmid profile, revealed by autoradiography, using ${ }^{32}$ P-labelled pK18mob as probe. Chr, chromosome. c PCR products of three different strains with a set of primers that distinguish among insertions for the LP sector in the chromosome (lanes C, primers ChrleftD and ChrightD), or pRetCFN42a (lanes a, primers LwattA1 and M13 reverse) and pRetCFN42d (lanes d, primers LwattD1 and M13 reverse)

To further explore the stability of integrants on the LP sector, flow cytometry analyses were carried out. In these experiments, $10^{7}$ cells derived from serial subcultures in the absence of antibiotics (21 generations) were screened for fluorescence, thus constituting a more robust assay. As shown in Fig. 5a, most of the cells corresponding to the insertion of the LP sector on the chromosome display strong fluorescence. Only about 100 cells $\left(1 \times 10^{-5}\right)$ in this population failed to display fluorescence, but these appear to be mainly young cells. In contrast, in a population of $10^{7}$ cells containing the $\mathrm{pK} 18 \mathrm{mob}$ att $\Delta$ plac integrated in the LP sector, all the cells failed to
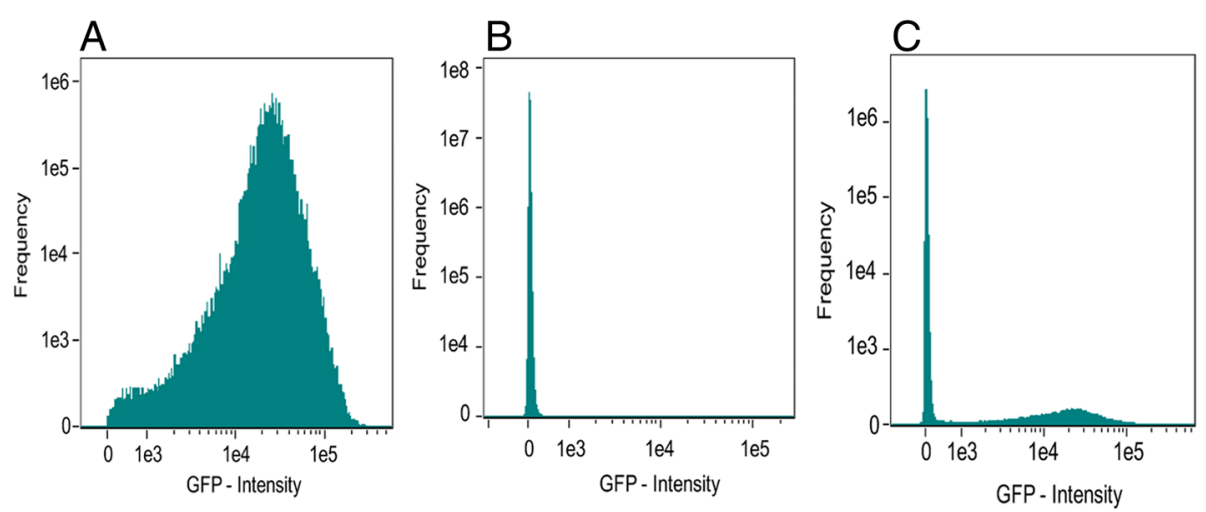

Fig. 5 Flow cytometry analyses. For each sample, data from a minimum of $10^{7}$ cells serially subcultured in the absence of antibiotics, for a period representing 21 generations were collected using ImageStream X imaging flow cytometer and analyzed using IDEAS 5.0 software. a cells with insertion of the LP sector on the chromosome display strong fluorescence. $\mathbf{b}$ cells with pK18 mob att $\Delta$ plac inserted in the LP sector. $\mathbf{c}$ Mixture of cells corresponding to integration of pK18 mob att $\Delta$ plac in the LP sector $\left(10^{7}\right.$ cells) and the strain harboring the LP sector ( $10^{3}$ cells) 
display fluorescence, (Fig. 5b). To verify that this assay can reliably identify fluorescent cells out of a large population of nonfluorescent cells, $10^{7}$ cells with pK18 mob att $\Delta$ plac integrated in the LP sector were mixed with $10^{3}$ fluorescent cells corresponding to the strain harboring the LP sector. As shown in Fig. 5c, flow cytometry analysis clearly reveals two populations differing in fluorescence, in the expected proportions. Overall, the results presented above confirm that the insertions on the LP sector are highly stable.

After demonstrating that integration may occur on the LP sector, we then asked if integration on the LP sector still leaves it susceptible to further integration events. Integrants on the LP sector possess two att sites separated by the inserted DNA. These integrants are nonfluorescent due to the integration of sequences lacking an active promoter for transcription of the GFP gene. We reasoned that further integration of a sequence harboring an active promoter on the rightmost att site (i. e. the one closer to the GFP gene) should restore GFP production.

To explore this, we took advantage of the previously built Bordetella suicide plasmid pG18mob $\left(\mathrm{Gm}^{\mathrm{r}}\right)$ [14]. This plasmid lacks any detectable sequence similarity with pK18 mob att $\Delta$ plac, other than the mob segment, and it possesses a placZ promoter adjacent to the MCS sequence. Into the MCS sequence of this plasmid, an att fragment was cloned as an EcoRI-SalI fragment (see Methods and Fig. 6b). After conjugative transfer of pG18 $m o b$ att to the $R$. etli LP strain that already contained an integrated pK18 mob att $\Delta$ plac, $\mathrm{Km}^{\mathrm{r}} \mathrm{Gm}^{\mathrm{r}}$ integrants were detected at a frequency of $1.8 \times 10^{-4}$ per recipient cell. A total of 261 integrants (from two independent experiments) were analyzed for GFP expression; 8 (3.06 \%) of them recovered fluorescence production. These results are compatible with further insertions on the LP sector. These integrations may have occurred on the att sequence located either at the left side or the right side of the LP sector (Fig. 6c). We reasoned that insertions on the right side were most likely, since they place a plac $Z$ promoter in proximity to the GFP gene, thus explaining their fluorescent nature.

To verify the location of additional insertions within the LP sector, specific PCR assays were set up. These PCR assays comprised two sets of primers; the first one included a primer complementary to the left side of the LP sector (ChrleftD) and the other matched the sequence of pG18 mob att (G18lw) while the second pair had a primer complementary to the right side of the LP sector (ChrightD) and another to pG18 mob att (G18up). Amplification with the first set of primers revealed integration on the left att sequence, while PCR products obtained with the second set reveals integration on the right att sequence (Fig. 6c). As expected, four of the integrants characterized revealed amplification with the second set of primers but not with the first set, indicating that integration indeed occurred on the rightmost att site (Fig. 6d). Interestingly, one of the fluorescent derivatives gave positive amplification with both primer sets, indicating the possibility of multiple integration events on the LP sector (Fig. 6d).

\section{Conclusions}

As shown in this work, site-specific bacterial chromosome engineering mediated by IntA allows the efficient integration of sequences lacking extensive homology to the host genome, other than the att sequence. Use of this approach requires a specially-constructed strain, harboring the so-called Landing Pad, containing a single att region, as well as GFP to allow easy identification of strains harboring the desired integration. The size of the construct to be integrated is limited only by the size of DNA that can be successfully cloned into the donor vector via ligation. Transfer of foreign DNA into the LP strain is done by conjugation, thus circumventing the need for DNA purification and transformation, making it a very robust approach. Under appropriate conditions, integrants were derived at a high frequency $\left(10^{-4}\right)$ compared to the frequency of double crossover recombination $\left(10^{-6}\right)$. The integrated DNA is flanked by copies of the att sequences, which are potential targets for IntA-mediated excision. Nevertheless, integrants are very stable, due to the low frequency of excision, evaluated previously at $2.1 \times 10^{-6}$ [8]. In fact, in the experiments reported here, no IntA-mediated excision events were detected. The finding that the att sequences added by integration are still active for recombination, open up the possibility of larger, more complex alternatives of strain construction in a sequential way.

Although this is not the first system described that allows integration mediated by site-specific recombination, it offers some advantages compared to other systems. For instance, recombineering via $\lambda$-red, a phage based system, requires larger homologous segments, and has limited effectiveness in bacteria other than E. coli [15] The ФC31 integrase has a different recombination target than IntA, and it has demonstrated efficient activity in disparate hosts, including Sinorhizobium meliloti [16]. IntA has the advantages of efficiency and modularity, given that any donor cassette can potentially be integrated into any LPstrain. In this regard, several rhizobial species possess IntA-homologs, with conceivably different specificities. Ideally, the IntA system may be combined with other site-specific systems with different specificity, to facilitate bacterial genome engineering. 


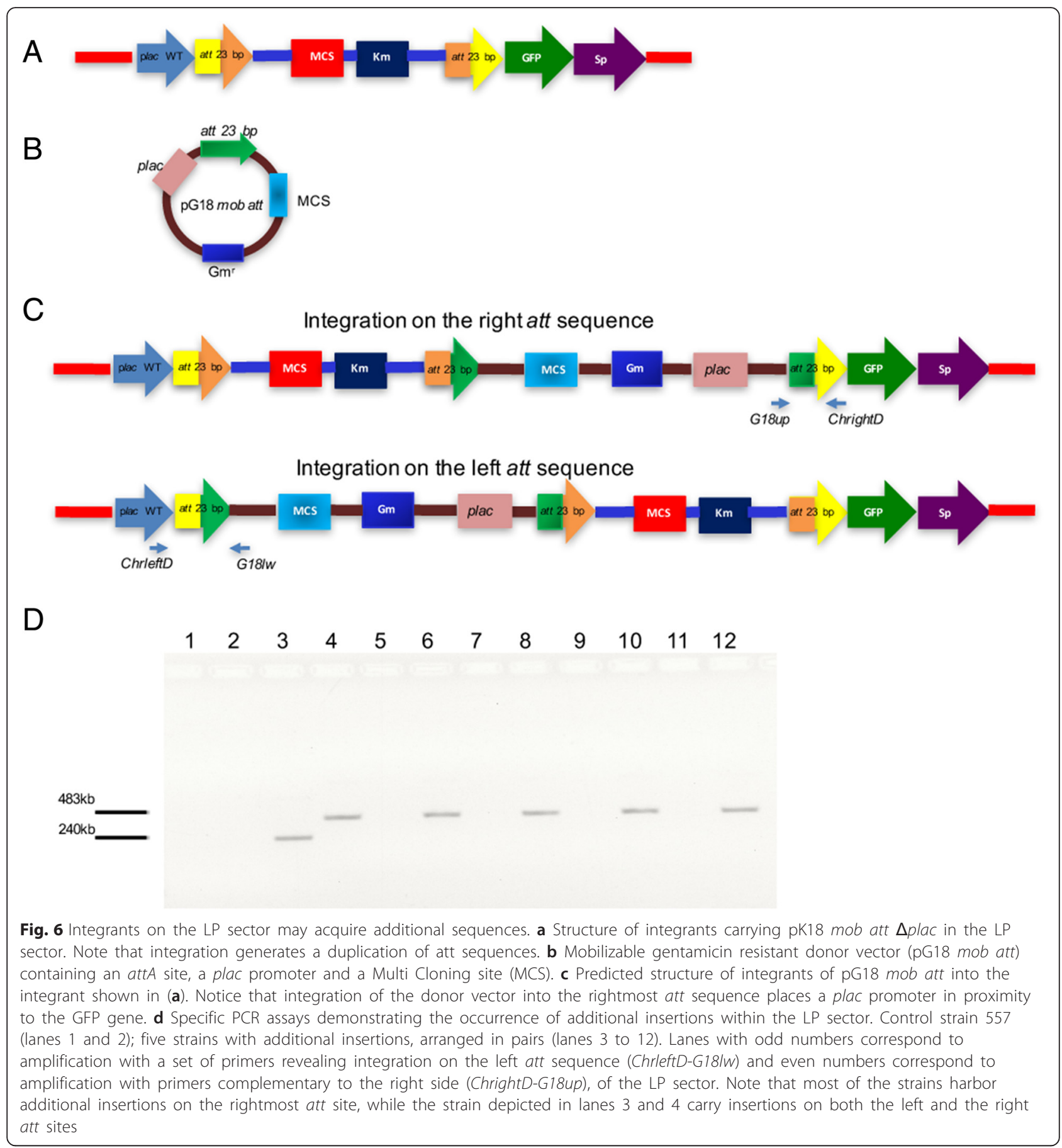

\section{Methods}

\section{Bacterial strains, plasmids and growth conditions}

Rhizobium strains were grown at $30{ }^{\circ} \mathrm{C}$ in PY (peptone-yeast extract) medium [17]. Escherichia coli strains were grown at $37{ }^{\circ} \mathrm{C}$ in LB (Luria-Bertani) medium [18]. When needed, antibiotics were added at the following concentrations (in micrograms per milliliter): nalidixic acid, 20; spectinomycin, 100; kanamycin, 30.

\section{Biparental mating}

Plasmid transfer from E. coli to Rhizobium was done by biparental mating, using E. coli S17.1 harboring the suicide vector (pK18 mob att $\Delta$ plac) as a donor and $R$. etli 557 (LP-strain) as recipient, the strains were grown in liquid medium to stationary phase, washed twice with PY medium, mixed in a donor/recipient ratio of 1:2 on PY plates, and incubated at $30{ }^{\circ} \mathrm{C}$ overnight. After incubation, cells were resuspended in $\mathrm{MgSO}_{4} 10$ mM-Tween 
$0.01 \%$, serially diluted and plated on solid PY medium containing nalidixic acid, spectinomycin and kanamycin. Cointegration frequencies were evaluated independently at least thrice, and are expressed as number of transconjugants per recipient cell. For evaluation of GFP production, colonies were visualized under ultraviolet light (425 nm).

\section{Molecular and microbiological procedures}

Basic DNA manipulations and molecular techniques were done using established procedures [19]. Extraction of DNA from agarose gels was done with a GeneJET extraction kit (Fermentas); plasmids were isolated with high pure plasmid isolation kit (Roche). All oligonucleotides used were synthesized at the Unidad de Síntesis of the Instituto de Biotecnología, Universidad Nacional Autónoma de México; all PCR amplifications were carried out using High Fidelity Taq polymerase (Invitrogen). Amplification protocols consisted of 30 cycles of $1 \mathrm{~min}$ at $94{ }^{\circ} \mathrm{C}, 1 \mathrm{~min}$ at variable temperature (depending on the primer combination), and 1 to $3 \mathrm{~min}$ at $68{ }^{\circ} \mathrm{C}$. After amplification, PCR products were extracted with phenol and precipitated with ethanol. The DNAs were resuspended in Tris-EDTA buffer and digested with the appropriate restriction enzyme(s) to generate the required ends in the fragments. The DNA fragments were purified before cloning by isolating them from the agarose gel. For ligations, T4 polynucleotide ligase (Fermentas) was used. Plasmid transformation of E. coli was done using $\mathrm{CaCl}_{2}$-competent cells. All plasmid constructions were verified by restriction analysis and PCR and, in most of the cases, by DNA sequencing.

\section{Plasmid construction}

To generate the LP sector, a cassette containing a green fluorescent protein gene under the control of the lacZ promoter and a spectinomycin resistance gene [10] was modified by the introduction of an att site. To do that, the attA site was amplified from $R$. etli genomic DNA using primers UpattA1 and LwattA1, gel-purified and restricted with EcoRI and BamHI and ligated into similarly restricted $\mathrm{pCR} 2.1^{\circ}-\mathrm{TOPO}^{\circ} \mathrm{TA}$ vector (Thermo Fisher Scientific) From the resulting plasmid, the attA sequence was excised by digestion with $\mathrm{Xba \textrm {I }}$ and ligated into XbaI-digested pGX534 [10], giving rise to plasmid pRG10. In this plasmid, the lacZ promoter and the GFP gene are separated by an att sequence. To facilitate insertion of the LP sector in the chromosome, a $477 \mathrm{bp}$ chromosomal segment (from nucleotide 1108084 to nucleotide 1108560 , corresponding to the non-coding region) was amplified using primers chr_left_in_hind and chr_right_in_eco (Table 1). This PCR fragment was digested with HindIII and EcoRI and cloned into similarly digested pK18mob-sacB [20] yielding pGX117. To substitute a single PstI site present within the noncoding region with a NotI site, pGX117 was digested with PstI and ligated to oligonucleotide Link-PstxNot, giving rise to plasmid pGX191.

To generate a chromosomal entry vector for the LP sector, plasmid pRG10 was digested with NotI (thus excising a NotI cassette harboring the LP sector), and the resulting fragment was cloned into NotI digested pGX191. The resulting chromosomal entry vector for the LP sector was called pRG11.

To generate a plasmid useful for integration in the LP sector, a derivative of plasmid pK18 mob [20], harboring an $a t t D$ sequence and lacking the native plac promoter was constructed. To do that, two complementary oligonucleotides (Lw23bp and Up23bp, Table 1), containing the $a t t D$ sequence, were heat-denatured and reannealed, and the resulting double-stranded fragment was restricted with $B a m H I$. This product was ligated into similarly restricted pK18 mob, giving rise to pK18 mob att. To remove a $160 \mathrm{bp}$ segment containing the lac promoter, pK18 mob att was restricted with AseI and EcoRI, ends were filled-in with Klenow polymerase and the plasmid was religated in a blunt-end ligation, resulting in plasmid pK18 mob att $\Delta$ plac $(3.66 \mathrm{~kb})$.

A separate vector, useful to investigate the possibility of repeated insertions into an already occupied LP sector (plasmid pG18 mob att) was also constructed. To this end, the attA site was amplified from $R$. etli genomic DNA using primers UpattA1 and LwattA1, gel-purified and restricted with EcoRI and BamHI and ligated into similarly restricted pG18 mob [14], thus generating pG18 mob att (2.88 kb).

\section{Plasmid profiles and hybridization}

Rhizobium plasmids were visualized by the Eckhardt procedure [12]. Gels were transferred onto Hybond $\mathrm{N}^{+}$membranes (Amersham) using the manufacturer's protocol and cross-linked using a UV cross linker unit (Stratagene). Hybridizations were performed overnight using $\alpha^{32} \mathrm{P}$ dCTP-labelled probes (Megaprime kit; Amersham) under high-stringency conditions $\left(65{ }^{\circ} \mathrm{C}\right.$ in rapid-Hyb buffer, Amersham). Hybridization signals were detected with a PhosphorImager (Molecular Dynamics).

\section{Flow cytometry}

For determination of stability of the integrated DNA by flow cytometry, cells from the desired strain were subjected to three serial overnight cultures in the absence of antibiotics (representing about 21 generations) and analyzed by flow cytometry. Cells were analyzed in the ImageStream X imaging flow cytometer (Amnis Corporation, Seattle, WA) using the $488 \mathrm{~nm}$ solid-state laser with appropriate compensation controls and settings. Data were acquired using INSPIRE acquisition software and the 60X 
objective lens on a low speed setting. Data from a minimum of $10^{7}$ cells were collected for each sample and analyzed using IDEAS 5.0 software. The instrument and INSPIRE software were set up as follows: Channel 01 (bright field), channel 02 (green fluorescence) and channel 12 (scattering channel). Magnification was $60 \mathrm{X}$, providing a pixel size of $0.3 \mu \mathrm{m} 2$ and the lasers 488 and 745 activated for fluorescence and side-scatter, respectively. The flow rate was set to low speed/high sensitivity and stream alignment was adjusted where necessary. Focused images of single cells were initially identified from the brightfield images taken by the imaging flow cytometer by using the manufacturer's recommended analysis algorithm, the gradient root mean square feature. To reduce artifacts and background noise, we used the default mask of the IDEAS 5.0 software called M01 and an investigator-defined mask, which was composed of a combination of mask features (system 50, erode 2, range 300-2,000). Areas of interest smaller than 300 pixels were deselected. Images that still had a spot count of two or more, were excluded and designated as "doublets."

\section{Abbreviations}

${ }^{\circ} \mathrm{C}$, celsius degree; att, attachment site; attA, attachment site plasmid a; attD, attachment site plasmid d; Bp, base pairs; Chr, chromosome; DNA, deoxyribonucleic acid; EDTA, ethylenediaminetetraacetic acid; GFP, green fluorescent protein; $\mathrm{Gm}^{r}$, gentamicin resistant; LB, luria-bertani; LP, landing pad; MCS, multi cloning site; Nm, nanometer; PCR, polymerase chain reaction; PY, peptone-yeast extract; UV, ultraviolet.

\section{Acknowledgements}

We gratefully acknowledge Laura Cervantes and Araceli Dávalos for skillful technical assistance, Paul Gaytán and Eugenio López (Unidad de Síntesis de Oligonucleótidos, Instituto de Biotecnología, UNAM) for help with oligonucleotide synthesis and Andres Saralegui Amaro (Laboratorio Nacional de Microscopía Avanzada, Instituto de Biotecnología, UNAM) for help with flow cytometry experiments. G.T-T is member of the Research Career of CONICET, Argentina.

\section{Funding}

The study was supported with internal funds from the Centro de Ciencias Genómicas, Universidad Nacional Autónoma de México.

\section{Availability of data and materials}

The data that support the results of the present study are included within the journal article.

\section{Authors' contributions}

$\mathrm{RH}-\mathrm{T}, \mathrm{GT}-\mathrm{T}, \mathrm{SB}$ and DR conceived and designed the experiments; $\mathrm{RH}-\mathrm{T}$ and GT-T carried out the experiments; RH-T, GT-T, SB and DR analyzed the data; $\mathrm{RH}-\mathrm{T}$ and DR wrote the paper. All authors read and approved the final version of the manuscript.

\section{Competing interests}

The authors declare that they have no competing interests.

\section{Consent for publication}

Not applicable.

\section{Author details}

${ }^{1}$ Programa de Ingeniería Genómica, Centro de Ciencias Genómicas, Universidad Nacional Autónoma de México, Apartado Postal 565-A, 62210 Cuernavaca, Morelos, Mexico. ${ }^{2}$ Departamento de Ciencias Biológicas, Instituto de Biotecnología y Biología Molecular, UNLP, CCT-La Plata-CONICET, Facultad de Ciencias Exactas, Universidad Nacional de La Plata, La Plata, Argentina.

Received: 16 March 2016 Accepted: 28 June 2016

Published online: 29 June 2016

References

1. Hallet B, Sherratt DJ. Transposition and site-specific recombination: adapting DNA cut-and-paste mechanisms to a variety of genetic rearrangements. FEMS Microbiol. 1997;21:157-78.

2. Ghosh K, Lau CK, Gupta K, Van Duyne GD. Preferential synapsis of loxP sites drives ordered strand exchange in Cre-loxP site-specific recombination. Nat Chem Biol. 2005;1:275-82.

3. Atlung T, Nielsen A, Rasmussen $L$, Nellemann $L$, Holm FA. Versatile method for integration of genes and gene fusions into the $\lambda$ attachment site of Escherichia coli. Gene. 1991;107:11-7.

4. Lee CY, Buranen SL, Zhi-Hai Y. Construction of single-copy integration vectors for Staphylococcus aureus. Gene. 1991;103:101-5.

5. Lee MH, Pascopella L, Jacobs WR, Hatfull GF. Site-specific integration of mycobacteriophage L5: integration-proficient vectors for Mycobacterium smegmatis, Mycobacterium tuberculosis, and bacille Calmette-Guérin. Proc Natl Acad Sci U S A. 1991:88:3111-5.

6. Smokvina T, Mazodier P, Boccard F, Thompson CJ, Guérineau M. Construction of a series of pSAM2-based integrative vectors for use in actinomycetes. Gene. 1990;94:53-9.

7. Loots GG. Modifying yeast artificial chromosomes to generate Cre/LoxP and FLP/ FRT site-specific deletions and inversions. Methods Mol Biol. 2006;349:75-84.

8. Hernández-Tamayo R, Sohlenkamp C, Puente JL, Brom S, Romero D. Characterization of IntA, a bidirectional site-specific recombinase required for conjugative transfer of the symbiotic plasmid of Rhizobium etli CFN42. J Bacteriol. 2013;195:4668-77.

9. Brom S, Girard L, Tun-Garrido C, Garcia-De Los Santos A, Bustos P, González $\checkmark$, Romero D. Transfer of the symbiotic plasmid of Rhizobium etli CFN42 requires cointegration with $\mathrm{p} 42 \mathrm{a}$, which may be mediated by site-specific recombination. J Bacteriol. 2004;186:7538-48.

10. Tejerizo GT, Bañuelos LA, Cervantes L, Gaytán P, Pistorio M, Romero D, Brom S. Development of molecular tools to monitor conjugative transfer in rhizobia. J Microbiol Methods. 2015;117:155-63.

11. López-Leal G, Tabche ML, Castillo-Ramírez S, Mendoza-Vargas A, RamírezRomero MA, Dávila G. RNA-Seq analysis of the multipartite genome of Rhizobium etli CE3 shows different replicon contributions under heat and saline shock. BMC Genomics. 2014;15:770.

12. Eckhardt T. A rapid method for the identification of plasmid desoxyribonucleic acid in bacteria. Plasmid. 1978;1:584-8.

13. Hynes MF, Simon R, Müller P, Niehaus K, Labes M, Pühler A. The two megaplasmids of Rhizobium meliloti are involved in the effective nodulation of alfalfa. Mol Gen Genet. 1986;202:356-62.

14. Kirchner O, Tauch A. Tools for genetic engineering in the amino acidproducing bacterium Corynebacterium glutamicum. J Biotechnol. 2003; 104:287-99.

15. Westenberg M, Bamps S, Soedling H, Hope IA, Dolphin CT. Escherichia coli MW005: lambda Red-mediated recombineering and copy-number induction of oriV-equipped constructs in a single host. BMC Biotechnol. 2010. doi:10. 1186/1472-6750-10-27.

16. diCenzo G, Milunovic B, Cheng J, Finan TM. The tRNAarg gene and engA are essential genes on the 1.7-Mb pSymB megaplasmid of Sinorhizobium meliloti and were translocated together from the chromosome in an ancestral strain. J Bacteriol. 2013; doi: 10.1128/JB.01758-12.

17. Noel KD, Sánchez A, Fernández L, Leemans J, Cevallos MA. Rhizobium phaseoli symbiotic mutants with transposon Tn5 insertions. J Bacteriol. 1984;158:148-55.

18. Hanahan D. Studies on transformation of Escherichia coli with plasmids. J Mol Biol. 1983;166:557-80.

19. Sambrook J, Fritsch EF, Maniatis T. Molecular cloning: a laboratory manual. 2nd ed. New York: Cold Spring Harbor Laboratory; 1989.

20. Schäfer A, Tauch A, Jäger W, Kalinowski J, Thierbach G, Pühler A. Small mobilizable multi-purpose cloning vectors derived from the Escherichia coli plasmids pK18 and pK19: selection of defined deletions in the chromosome of Corynebacterium glutamicum. Gene. 1994;145:69-73. 